\title{
Avaliando Conhecimento em DST de Graduandos em Medicina segundo a Taxonomia de Bloom
}

\author{
Evaluating Medical Students' Knowledge on \\ STI According to Bloom's Taxonomy
}

PALAVRAS-CHAVE:

- Educação Médica;

- Doenças Sexualmente Transmissíveis;

- Estudantes de Medicina;

- Avaliação Educacional.

\section{KEYWORDS}

- Medical Education;

- Sexually Transmitted Infections;

- Medical Students;

- Educational Assessment.
Throughout a medical degree and its students' gradual entry into an area of specialization, cognitive assessments are widely applied and used in order to analyze learning procedures. The instrument used is not however always compatible or effective in evaluating the topics studied. Apart from gauging the extent of medical students' knowledge of STIs, the aim of this study is to investigate the existence of preferential distributions with regard to gender. In this descriptive observational cross-sectional study, 25 multiple-choice tests were, formulated according to Bloom's typology, the degree of difficulty and STIs' thematic core. They were then applied to 68 sixth-year interns. Results registered preferential distribution $(p<0.001)$ with homogeneity of students in the direction of the most frequent alternative and a polarization of correct answers in the categories of Knowledge and Analysis. The findings are representative of the current medical education model, in which the transmission of information dominates a development and reconstruction of knowledge, leading to the search for new strategies in teaching and assessment.

Eloá Rondi Bórnea
Aguinaldo Gonçalves Carlos Roberto Padovani ${ }^{I I}$

Durante a graduação e o ingresso na especialidade, as avaliações cognitivas são amplamente aplicadas e utilizadas para analisar o aprendizado. Entretanto, nem sempre o instrumento empregado é compatível e suficiente para avaliar os tópicos aprendidos. O objetivo da presente investigação, além de apreender o grau de conhecimento que graduandos de Medicina detêm sobre DST, é investigar a existência de distribuições preferenciais a respeito segundo o gênero. Trata-se de um estudo observacional transversal descritivo de retroanálise. Foi adotada uma bateria de 25 testes de múltipla escolha formulados segundo a tipologia de Bloom, grau de dificuldade, núcleo temático das DST e aplicados a 68 internos do sexto ano. Os resultados registraram distribuição preferencial $(p<0,001)$ com homogeneidade do alunado em direção à alternativa mais frequente e polarização dos acertos nas categorias Conhecimento e Análise. Esses dados ilustram o modelo de educação médica atual, em que predomina a transmissão de informações ao invés da elaboração e reconstrução do conhecimento, o que tem levado à busca de novas estratégias de ensino e avaliação. 


\section{INTRODUÇÃO}

Segundo as Diretrizes Curriculares Nacionais, os cursos de Ciências Médicas identificam-se pelo objetivo de formar profissionais com conhecimento cognitivo e habilidades para atuação na saúde individual e coletiva ${ }^{1}$. No entanto, com estruturas curriculares rígidas, constroem certa dicotomia entre a teoria e a prática, tornando a residência médica uma extensão para o desenvolvimento da atividade clínica ${ }^{2}$. Durante a graduação e o ingresso na especialidade, as avaliações cognitivas são amplamente aplicadas e utilizadas para analisar o aprendizado. Entretanto, nem sempre o instrumento empregado é compatível e suficiente para avaliar os tópicos aprendidos.

Estudando as formas e aplicações de questionários, Case e Swason ${ }^{3}$ expressaram orientações para a criação de testes capazes de analisar o conhecimento médico. Destacaram que a estratégia de "verdadeiro ou falso" seria o mecanismo menos eficiente para esse objetivo porque as sentenças, por não estarem contextualizadas, não se mostram claras, podendo ser parcialmente corretas ou incorretas, sem mencionar os cinquenta por cento de chance de acerto apenas com um palpite. Dessa maneira, ganham força os testes de múltipla escolha, já que é possível construir casos clínicos sobre o tema selecionado e solicitar que entre cinco opções o estudante escolha a que melhor se aplique.

Além desses modelos, outra técnica é a de associação, em que se toma um tema, criam-se diversas opções pertinentes e apresentam-se diferentes casos clínicos, diante do que o candidato deve selecionar a opção que melhor se relacione às situações-problema ilustradas. Essa forma, quando comparada à de alternativas, demonstrou maior poder discriminativo. $\mathrm{A}$ justificativa é que, em questões de associação, o candidato é exposto a diferentes circunstâncias e variadas respostas, sendo necessário correlacionar às informações a identificação do paciente, sinais e sintomas, exame físico e exames complementares para conseguir encontrar uma ou mais respostas que se adaptem à realidade exposta, o que exige raciocínio complexo e verifica a possibilidade de detecção de erros quando o assunto não está bem sedimentado ${ }^{4}$

No Brasil, o Instituto Nacional de Educação e Pesquisa Júlio de Mesquita (Inep) é o órgão público responsável por criar os mecanismos para validar e classificar os cursos de ensino superior, dentre os quais o de Medicina, por meio do Exame Nacional de Desempenho do Estudante (Enade). Apesar da existência de novos meios de verificações, os testes de múltipla escolha continuam sendo utilizados, por permitirem, com as vantagens de rapidez e objetividade na correção e menor vulnerabilidade a erros de julgamentos do corretor, verificar tanto tarefas simples de memorização e reconhecimento, quanto complexas, de compreensão, aplicação, síntese e avaliação a partir de situações-problemas ${ }^{5}$.

Esta sistematização dos domínios cognitivos se deve a Benjamin Bloom, que criou uma classificação hierárquica dos níveis de aprendizagem, que busca meios de estimular, nos estudantes, raciocínio e abstraçoes de alto padrão. Isto se deve à existência de uma relação de dependência entre as categorias, que são organizadas em termos da complexidade dos processos mentais, sendo a primeira o pensamento mais básico, e a última, o mais elaborado ${ }^{6}$.

Além disso, para um teste de qualidade deve-se considerar o nível de dificuldade da pergunta aplicada - fácil, média e difícil -, de acordo com a complexidade da informação escolhida. No caso de provas médicas, procura-se evitar conhecimentos estritamente especializados, pretendendo-se abordar conceitos fundamentais para uma atuação profissional coerente ${ }^{7}$.

O presente projeto relata diferentes aspectos decorrentes da experiência de aplicação de uma bateria de questões de múltipla escolha a estudantes do sexto ano de Medicina sobre doenças sexualmente transmissíveis. Além de apreender o grau de conhecimento que detêm sobre esse grupo de agravos no interior da mencionada escala de Bloom, interessa-nos investigar a existência de distribuições preferenciais a respeito segundo o gênero.

\section{METODOLOGIA}

Trata-se de um estudo observacional transversal descritivo de retroanálise, realizado durante o internato em Medicina da Pontifícia Universidade Católica de Campinas (PUC-Campinas), no ingresso à disciplina MED01622 - "Especialidades Clínicas Ambulatoriais: Dermatologia", trigésima oitava turma, décimo segundo período 8 .

Os dados foram obtidos em grupos de cinco a seis internos, semanas e meses antes da formatura, totalizando 68 unidades observacionais. Logo ao término, foi realizada a correção com a discussão dos erros e dúvidas.

Como instrumento de coleta foi utilizada uma folha de questões contendo uma bateria de 25 testes de múltipla escolha, acompanhada da respectiva folha de resposta. A formulação das questões pretendeu envolver a distribuição segundo as capacidades cognitivas da tipologia de Bloom, grau de dificuldade e núcleo temático das DST, conforme indicação do Quadro 1.

O processamento informatizado dos dados foi realizado pela aplicação do Software SPSS (Statistical Package for the Social Sciences). Os resultados descritivos, relativos à distribuição de gêneros entre os alunos e proporção de acertos e erros de cada candidato, são apresentados sob forma tabular, ${ }^{9}$. 
QuADRO 1

Distribuição das questões quanto ao núcleo temático de DST, capacidades cognitivas de Bloom e nível de dificuldade

\begin{tabular}{|c|c|c|c|c|}
\hline Variável & $\begin{array}{l}\text { Categoria de } \\
\text { resposta }\end{array}$ & $\begin{array}{l}\text { No de } \\
\text { questões }\end{array}$ & $\begin{array}{c}\text { Porcen- } \\
\text { tagem }\end{array}$ & Exemplos de questões \\
\hline \multirow{3}{*}{ Núcleo Temático } & Epidemiologia & 13 & 52,00 & \multirow{3}{*}{$\begin{array}{l}\text { Epidemiologia: O fator de risco mais importante para contração das } \\
\text { DST é: } \\
\text { a) atividade sexual intensa; } \\
\text { b) grande rotatividade de parceiro; } \\
\text { c) maior mobilidade social; } \\
\text { d) condição de portadora assintomática. } \\
\text { Resposta certa: b. }\end{array}$} \\
\hline & $\begin{array}{l}\text { Clínica \& } \\
\text { Terapêutica }\end{array}$ & 7 & 28,00 & \\
\hline & Profilaxia & 5 & 20,00 & \\
\hline \multirow{6}{*}{$\begin{array}{c}\text { Capacidade } \\
\text { Cognitiva }\end{array}$} & Conhecimento & 7 & 28,00 & \multirow{6}{*}{$\begin{array}{l}\text { Conhecimento: As DST são responsáveis por aproximadamente } \\
\text { metade de quais dos seguintes agravos perinatais: } \\
\text { a) conjuntivites; } \\
\text { b) deficiências cognitivas; } \\
\text { c) pneumonias; } \\
\text { d) meningites. Resposta certa: a. } \\
\text { Análise: Para as DST, de modo geral, constitui a melhor medida } \\
\text { preventivista: } \\
\text { a) educação sexual; } \\
\text { b) vigilância epidemiológica; } \\
\text { c) disponibilidade de serviços de saúde; } \\
\text { d) vacinação dos suscetíveis. } \\
\text { Resposta certa: c. }\end{array}$} \\
\hline & Compreensão & 2 & 8,00 & \\
\hline & Aplicação & 2 & 8,00 & \\
\hline & Análise & 8 & 32,00 & \\
\hline & Síntese & 3 & 12,00 & \\
\hline & Avaliação & 3 & 12,00 & \\
\hline \multirow{3}{*}{$\begin{array}{l}\text { Nível de } \\
\text { dificuldade }\end{array}$} & Fácil & 9 & 36,00 & \multirow{3}{*}{$\begin{array}{l}\text { Fácil: Trata-se de dificuldade relevante nos programas de Educação } \\
\text { Sanitária contra as DST: } \\
\text { a) tempo de incubação arrastado da moléstia; } \\
\text { b) correspondente a quadro clínico extremamente amplo; } \\
\text { c) extrema variabilidade intraespecífica dos agentes das gonococias; } \\
\text { d) natureza absolutamente pessoal e íntima dos comportamentos } \\
\text { relativos à sexualidade. } \\
\text { Resposta certa: d } \\
\text { Médio: A primeira opção terapêutica para uretrite por Chlamydia é: } \\
\text { a) tetraciclina; } \\
\text { b) doxiciclina; } \\
\text { c) eritromicina; } \\
\text { d) aminociclina. } \\
\text { Resposta certa: b. } \\
\text { Difícil: Para a afirmação: "Embora os registros vitais de morbidade, } \\
\text { de modo geral e sobretudo nos países terceiro-mundistas, sejam } \\
\text { de precária qualidade, os referentes às DST constituem expressiva } \\
\text { exceção PORQUE se trata de grupo de doenças de considerável } \\
\text { magnitude", responda: } \\
\text { a) se asserção e razão estiverem corretas; } \\
\text { b) se asserção e razão estiverem erradas; } \\
\text { c) se a asserção estiver correta e a razão errada; } \\
\text { d) se a asserção estiver errada e a razão correta. } \\
\text { Resposta certa: d. }\end{array}$} \\
\hline & Médio & 8 & 32,00 & \\
\hline & Difícil & 8 & 32,00 & \\
\hline
\end{tabular}

Além disso, o estudo também comporta abordagem analítica, para a avaliação das proporções de acertos/erros de cada questão. Nos estudos das associações entre os atributos e as categorias de respostas foi utilizado o teste de homogeneidade de Goodman, complementado com contrastes entre populações multinomiais ${ }^{11}$. Todas as inferências estatísticas foram realizadas ao nível de $5 \%$ de significância ${ }^{12}$.

\section{RESULTADOS}

A Tabela 1 apresenta a distribuição dos estudantes segundo o sexo. É possível observar um discreto predomínio do sexo feminino, assemelhando-se à proporção de gêneros observada na população em geral.

Na Tabela 2 tem-se a distribuição das respostas a cada uma das questões formuladas, registrando-se distribuição 
preferencial ( $\mathrm{p}<0,001$ ) em todas as questões, com exceção da número 17, o que indica homogeneidade do alunado em direção à alternativa mais frequente, entretanto ainda sem indicar se essa resposta é a julgada correta.

A Tabela 3 informa a distribuição das respostas segundo o sexo dos participantes, não sendo observada diferença estatisticamente significativa $(p<0,05)$ nas escolhas entre sexo feminino e masculino, exceto na questão 17.

As Tabelas 4 e 5 indicam a distribuição das respostas em termos de acertos e erros; esta, no plano mais geral, aponta auspiciosamente que os primeiros ocorreram três vezes mais que os segundos. Sob a ótica das categorias da tipologia de Bloom, no entanto, a polarização de frequência dos acertos se deu em duas categorias - conhecimento e análise - em 13 de 19 questões $(68,42 \%)$, enquanto os 31,57\% restantes se dispersaram nas três outras, não se registrando nenhum acerto na categoria de síntese.

TABELA 1

Distribuição absoluta e relativa dos alunos segundo o gênero

\begin{tabular}{lcc}
\hline \multicolumn{1}{c}{ Gênero } & No total & Porcentagem \\
\hline Feminino & 34 & 50,00 \\
Masculino & 31 & 45,58 \\
Não registrado & 3 & 4,41 \\
\hline Total & 68 & 100,00 \\
\hline
\end{tabular}

TABELA 2

Distribuição das respostas a cada uma das questões formuladas

\begin{tabular}{|c|c|c|c|c|c|}
\hline \multirow{2}{*}{$\begin{array}{c}\text { № } \\
\text { Questão }\end{array}$} & \multicolumn{4}{|c|}{ Respostas } & \multirow{2}{*}{ Total } \\
\hline & A & B & $\mathrm{C}$ & D & \\
\hline 1 & $0(0,0)$ & $48(73,9)$ & $0(0,0)$ & $17(26,1)$ & 65 \\
\hline 2 & $0(0,0)$ & $65(95,6)$ & $3(4,4)$ & $0(0,0)$ & 68 \\
\hline 3 & $3(4,4)$ & $4(5,9)$ & $56(82,4)$ & $5(7,4)$ & 68 \\
\hline 4 & $7(10,5)$ & $30(44,8)$ & $16(23,9)$ & $14(20,8)$ & 67 \\
\hline 5 & $2(3,0)$ & $14(21,2)$ & $6(9,1)$ & $44(66,7)$ & 66 \\
\hline 6 & $0(0,0)$ & $10(14,9)$ & $18(26,9)$ & $39(58,2)$ & 67 \\
\hline 7 & $2(2,9)$ & $54(79,4)$ & $11(16,2)$ & $1(1,5)$ & 68 \\
\hline 8 & $50(73,6)$ & $9(13,2)$ & $9(13,2)$ & $0(0,0)$ & 68 \\
\hline 9 & $18(26,5)$ & $5(7,4)$ & $44(64,6)$ & $1(1,5)$ & 68 \\
\hline 10 & $10(14,7)$ & $32(47,1)$ & $26(38,2)$ & $0(0,0)$ & 68 \\
\hline 11 & $24(36,4)$ & $5(7,6)$ & $15(22,7)$ & $22(33,3)$ & 66 \\
\hline 12 & $9(13,2)$ & $13(19,1)$ & $4(5,9)$ & $42(61,8)$ & 68 \\
\hline 13 & $15(22,4)$ & $9(13,4)$ & $38(56,7)$ & $5(7,5)$ & 67 \\
\hline 14 & $6(9,1)$ & $39(59,1)$ & $19(28,8)$ & $2(3,0)$ & 66 \\
\hline 15 & $24(36,4)$ & $25(37,8)$ & $11(16,7)$ & $6(9,1)$ & 66 \\
\hline
\end{tabular}

\begin{tabular}{|c|c|c|c|c|c|}
\hline \multirow{2}{*}{$\begin{array}{c}\text { № } \\
\text { Questão }\end{array}$} & \multicolumn{4}{|c|}{ Respostas } & \multirow{2}{*}{ Total } \\
\hline & A & B & $\mathrm{C}$ & D & \\
\hline $17^{*}$ & $11(16,4)$ & $13(19,4)$ & $23(34,3)$ & $20(29,9)$ & 67 \\
\hline 18 & $4(6,0)$ & $38(56,7)$ & $4(6,0)$ & $21(31,3)$ & 67 \\
\hline 19 & $3(4,6)$ & $45(68,1)$ & $15(22,7)$ & $3(4,6)$ & 66 \\
\hline 20 & $3(4,6)$ & $4(6,1)$ & $2(3,0)$ & $57(86,3)$ & 66 \\
\hline 21 & $58(85,3)$ & $4(5,9)$ & $2(2,9)$ & $4(5,9)$ & 68 \\
\hline 22 & $48(70,6)$ & $3(4,4)$ & $1(1,5)$ & $16(23,5)$ & 68 \\
\hline 23 & $26(39,4)$ & $3(4,6)$ & $19(28,8)$ & $18(27,2)$ & 66 \\
\hline 24 & $4(5,9)$ & $1(1,5)$ & $11(16,2)$ & $52(76,4)$ & 68 \\
\hline 25 & $26(50,0)$ & $5(9,6)$ & $13(25,0)$ & $8(15,4)$ & 52 \\
\hline
\end{tabular}

Tabela 3

Distribuição das respostas segundo o sexo dos participantes

\begin{tabular}{|c|c|c|c|c|c|c|}
\hline \multirow{2}{*}{\multicolumn{2}{|c|}{$\begin{array}{l}\text { Questões/ } \\
\text { Sexo }\end{array}$}} & \multicolumn{4}{|c|}{ Respostas } & \multirow{3}{*}{$\begin{array}{c}\text { Total } \\
33\end{array}$} \\
\hline & & \multirow{2}{*}{$\frac{\mathbf{A}}{0(0,0)}$} & \multirow{2}{*}{$\frac{\text { B }}{27(81,8)}$} & \multirow{2}{*}{$\frac{\text { C }}{0(0,0)}$} & \multirow{2}{*}{$\frac{\text { D }}{6(18,2)}$} & \\
\hline 1 & $\mathrm{~F}$ & & & & & \\
\hline & M & $0(0,0)$ & $19(65,5)$ & $0(0,0)$ & $10(34,5)$ & 29 \\
\hline \multirow[t]{2}{*}{2} & F & $0(0,0)$ & $33(94,3)$ & $2(5,7)$ & $0(0,0)$ & 35 \\
\hline & M & $0(0,0)$ & $29(96,7)$ & $1(3,3)$ & $0(0,0)$ & 30 \\
\hline \multirow[t]{2}{*}{3} & F & $2(5,7)$ & $0(0,0)$ & $31(88,6)$ & $2(5,7)$ & 35 \\
\hline & M & $1(3,3)$ & $4(13,3)$ & $22(73,3)$ & $3(10,0)$ & 30 \\
\hline \multirow[t]{2}{*}{4} & F & $4(11,8)$ & $16(47,1)$ & $6(17,6)$ & $8(23,5)$ & 34 \\
\hline & M & $2(6,7)$ & $13(43,3)$ & $9(30,0)$ & $6(20,0)$ & 30 \\
\hline \multirow[t]{2}{*}{5} & F & $2(5,9)$ & $11(32,4)^{*}$ & $3(8,8)$ & $18(52,9)$ & 34 \\
\hline & M & $0(0,0)$ & $2(6,9)$ & $3(10,3)$ & $24(82,8)$ & 29 \\
\hline \multirow[t]{2}{*}{6} & $\mathrm{~F}$ & $0(0,0)$ & $5(14,3)$ & $7(20,0)$ & $23(65,7)$ & 35 \\
\hline & M & $0(0,0)$ & $4(13,8)$ & $11(37,9)$ & $14(48,3)$ & 29 \\
\hline \multirow[t]{2}{*}{7} & F & $1(2,9)$ & $29(82,9)$ & $4(11,4)$ & $1(2,9)$ & 35 \\
\hline & M & $1(3,3)$ & $22(73,3)$ & $7(23,3)$, & $0(0,0)$ & 30 \\
\hline \multirow[t]{2}{*}{8} & $\mathrm{~F}$ & $24(68,6)$ & $4(11,4)$ & $7(20,0)$ & $0(0,0)$ & 35 \\
\hline & M & $23(76,7)$ & $5(16,7)$ & $2(6,7)$ & $0(0,0)$ & 30 \\
\hline \multirow[t]{2}{*}{9} & $\mathrm{~F}$ & $11(31,4)$ & $2(5,7)$ & $22(62,9)$ & $0(0,0)$ & 35 \\
\hline & M & $7(23,3)$ & $3(10,0)$ & $19(63,3)$ & $1(3,3)$ & 30 \\
\hline \multirow[t]{2}{*}{10} & F & $6(17,1)$ & $17(48,6)$ & $12(34,3)$ & $0(0,0)$ & 35 \\
\hline & M & $4(13,3)$ & $13(43,3)$ & $13(43,3)$ & $0(0,0)$ & 30 \\
\hline \multirow[t]{2}{*}{11} & F & $12(35,3)$ & $2(5,9)$ & $6(17,6)$ & $14(41,2)$ & 34 \\
\hline & M & $11(36,7)$ & $3(10,0)$ & $9(30,0)$ & $7(23,3)$ & 30 \\
\hline \multirow[t]{2}{*}{12} & F & $2(5,7)$ & $7(20,0)$ & $3(8,6)$ & $23(65,7)$ & 35 \\
\hline & M & $5(16,7)$ & $6(20,0)$ & $0(0,0)$ & $19(63,3)$ & 30 \\
\hline \multirow[t]{2}{*}{13} & $\mathrm{~F}$ & $7(20,6)$ & $4(11,8)$ & $19(55,9)$ & $4(11,8)$ & 34 \\
\hline & M & $8(26,7)$ & $4(13,3)$ & $17(56,7)$ & $1(3,3)$ & 30 \\
\hline \multirow[t]{2}{*}{14} & $\mathrm{~F}$ & $1(3,0)$ & $23(69,7)$ & $9(27,3)$ & $0(0,0)$ & 33 \\
\hline & M & $3(10,0)$ & $16(53,3)$ & $9(30,0)$ & $2(6,7)$ & 30 \\
\hline \multirow[t]{2}{*}{15} & $\mathrm{~F}$ & $11(32,4)$ & $11(32,4)$ & $8(23,5)$ & $4(11,8)$ & 34 \\
\hline & M & $11(37,9)$ & $13(44,8)$ & $3(10,3)$ & $2(6,9)$ & 29 \\
\hline \multirow[t]{2}{*}{16} & F & $21(60,0)$ & $11(31,4)$ & $1(2,9)$ & $2(5,7)$ & 35 \\
\hline & M & $18(60,0)$ & $12(40,0)$ & $0(0,0)$ & $0(0,0)$ & 30 \\
\hline
\end{tabular}




\begin{tabular}{|c|c|c|c|c|c|c|}
\hline \multirow{2}{*}{\multicolumn{2}{|c|}{$\begin{array}{l}\text { Questões/ } \\
\text { Sexo }\end{array}$}} & \multicolumn{4}{|c|}{ Respostas } & \multirow{3}{*}{$\begin{array}{c}\text { Total } \\
35\end{array}$} \\
\hline & & \multirow{2}{*}{$\begin{array}{c}\mathbf{A} \\
8(22,9)\end{array}$} & \multirow{2}{*}{$\begin{array}{c}\text { B } \\
8(22,9)\end{array}$} & \multirow{2}{*}{$\frac{\text { C }}{5(14,3)}$} & \multirow{2}{*}{$\frac{\text { D }}{14(40,0)}$} & \\
\hline $17^{*}$ & F & & & & & \\
\hline & M & $2(6,7)$ & $5(16,7)$ & $18(60,0)$ & $5(16,7)$ & 30 \\
\hline \multirow[t]{2}{*}{18} & $\mathrm{~F}$ & $2(6,7)$ & $20(57,1)$ & $2(5,7)$ & $11(31,4)$ & 35 \\
\hline & M & $2(6,9)$ & $16(55,2)$ & $2(6,9)$ & $9(31,0)$ & 29 \\
\hline \multirow[t]{2}{*}{19} & $\mathrm{~F}$ & $2(5,9)$ & $24(70,6)$ & $7(20,6)$ & $1(2,4)$ & 34 \\
\hline & M & $1(3,4)$ & $18(62,1)$ & $8(27,6)$ & $2(6,9)$ & 29 \\
\hline \multirow[t]{2}{*}{20} & $\mathrm{~F}$ & $2(5,9)$ & $3(8,8)$ & $0(0,0)$ & $29(85,3)$ & 34 \\
\hline & M & $1(3,4)$ & $1(3,4)$ & $1(3,4)$ & $26(89,8)$ & 29 \\
\hline \multirow[t]{2}{*}{21} & $\mathrm{~F}$ & $27(77,1)$ & $3(8,6)$ & $2(5,7)$ & $3(8,6)$ & 35 \\
\hline & M & $28(93,4)$ & $1(3,3)$ & $0(0,0)$ & $1(3,3)$ & 30 \\
\hline \multirow[t]{2}{*}{22} & $\mathrm{~F}$ & $27(77,1)$ & $3(8,6)$ & $1(2,9)$ & $4(11,4)$ & 35 \\
\hline & M & $19(63,3)$ & $0(0,0)$ & $0(0,0)$ & $11(36,7)$ & 30 \\
\hline \multirow[t]{2}{*}{23} & $\mathrm{~F}$ & $17(50,0)$ & $0(0,0)$ & $7(20,6)$ & $10(29,4)$ & 34 \\
\hline & M & $9(31,0)$ & $3(10,3)$ & $10(34,5)$ & $7(24,1)$ & 29 \\
\hline \multirow[t]{2}{*}{24} & $\mathrm{~F}$ & $2(5,7)$ & $0(0,0)$ & $9(25,7)$ & $24(68,6)$ & 35 \\
\hline & M & $2(6,7)$ & $1(3,3)$ & $2(6,7)$ & $25(83,3)$ & 30 \\
\hline \multirow[t]{2}{*}{25} & $\mathrm{~F}$ & $15(57,7)$ & $3(11,5)$ & $4(15,4)$ & $4(15,4)$ & 26 \\
\hline & M & $11(45,8)$ & $2(8,3)$ & $7(29,2)$ & $4(16,7)$ & 24 \\
\hline
\end{tabular}

${ }^{*} p<0,05$

TABELA 4

Medidas descritivas da distribuição de acertos praticados no instrumento utilizado

\begin{tabular}{c|c}
\hline Medidas descritivas & Valores observados \\
\hline Média & $13,33(53,35 \%)$ \\
Mediana & $14(56,00 \%)$ \\
Moda & $13(52,00 \%)$ \\
Valor máximo & $20(80,00 \%)$ \\
Valor mínimo & $6(24,00 \%)$ \\
\hline
\end{tabular}

TABELA 5

Distribuição dos acertos e erros nas respostas às questões formuladas, segundo as categorias da tipologia de Bloom

\begin{tabular}{lccccc}
\hline \multirow{2}{*}{ Categorias } & \multirow{2}{*}{ Total } & \multicolumn{2}{c}{ Acertos } & \multicolumn{2}{c}{ Erros } \\
\cline { 3 - 6 } & & No & \% & No & $\%$ \\
\hline Conhecimento & 7 & 7 & 100,00 & - & - \\
Análise & 8 & 6 & 75,00 & 2 & 25,00 \\
Compreensão & 2 & 1 & 50,00 & 1 & 50,00 \\
Aplicação & 2 & 2 & 100,00 & - & - \\
Síntese & 3 & - & - & 3 & 100,00 \\
Avaliação & 3 & 3 & 100,00 & - & - \\
\hline Total & 25 & 19 & 76,00 & 6 & 24,00 \\
\hline
\end{tabular}

\section{DISCUSSÃO}

Os dados apresentados mostram que, na matéria considerada, nossos alunos operam majoritariamente com conhecimento e análise, nada restando em termos de síntese. Como entender tais informações? Seriam especificidades diante dos aspectos das Doenças Sexualmente Transmissíveis ou característica mais geral do comportamento cognitivo desses jovens? Ou, num nível de maior generalização ainda, não seria sequer especificidade do grupo estudado, mas reflexo da sociedade e da educação do mundo em que vivemos?

Compatível com a exploração de aspectos da natureza dos mencionados acima, outro projeto de mesma orientação, realizado há poucos anos, aponta que, de modo geral, a educação no Brasil avança pouco, mantendo-se apenas como ferramenta instrucional, por meio de treinamentos, capacitações e transmissão de conhecimento. Ocorre a inoculação de informações de fora para dentro, não havendo espaço para processos elaborativos de reconstrução ${ }^{13}$.

Na área da educação médica, observa-se a necessidade de mudança, já que o curso de Medicina não consegue desenvolver o potencial intelectual, capacidade de análise, julgamento e avaliação crítica, habilidade para resolver problemas, raciocínio crítico com abordagem criativa do alunado. Diante das deficiências na graduação, fóruns nacionais e internacionais buscam a integralidade do ensino, com foco mais na saúde do que na doença, estimulando o papel ativo do aluno, além de capacitar os docentes tanto técnico-cientifica quanto pedagogicamente. Diante dessa realidade, a Comissão Interinstitucional de Avaliação de Ensino Médico (Cinaem), formada por 11 entidades representativas dos professores universitários, da profissão médica e de estudantes de Medicina do País, tem, durante a década de 1990, tentado introduzir novos métodos - entre eles o Problem Based Learning (PBL) e o Task Based Learning (TBL) - que melhorem o ensino e a formação desses profissionais ${ }^{14}$.

Em 1997, o PBL foi introduzido no Brasil com a intenção de interromper a lógica de acúmulo mecânico de informações, visando à construção do conhecimento por meio da resolução de problemas reais do futuro. Além da habilidade técnica e cognitiva, esse método pretende desenvolver atitudes mais aplicáveis tanto no cuidado do doente como na manutenção da postura de estudar para autoformação, incentivando a busca ativa do conhecimento ${ }^{15}$. Em termos aplicados, espera-se que seja mais eficaz que o método tradicional, visto que os acadêmicos lidam com relacionamentos interpessoais com melhor comunicação e atuação em equipes, com diferentes culturas e questões éticas, contribuindo para uma formação menos incompleta não apenas em nível intelectual, mas também nas dimensões sociais e pessoais. 


\section{CONCLUSÃO}

Os procedimentos executados na presente investigação permitiram conhecer as seguintes evidências:

- Em nosso meio, é exequível a avaliação de conhecimento profissional com base na taxonomia de Bloom;

— Em relação à bateria de 25 testes de múltipla escolha sobre Doenças Sexualmente Transmissíveis aplicada a graduandos de Medicina, observou-se, em termos de frequência, convergência de respostas, sem diferença expressiva entre os grupos de respondentes segundo o sexo;

- Ocorreram três vezes mais acertos do que erros, o que indica a prontidão desses jovens no manejo das situações apresentadas;

- Quando, porém, as respostas foram consideradas a partir da tipologia de Bloom, constatou-se predomínio de acertos em questões voltadas a aferir predominantemente Conhecimento e Análise (68,42\%), não se obtendo nenhum acerto na categoria Síntese;

- Tais resultados, indicativos de que os estudantes permanecem em níveis mais básicos da cognição, são interpretados no sentido de que na educação médica ainda predomina a transmissão de informações e conceitos com poucos estímulos à elaboração e construção da aprendizagem. Além da organização rígida do ensino, existe dificuldade dos docentes em exercer a busca ativa da reflexão.

\section{REFERÊNCIAS}

1. Goldwasser RS. A prova prática no processo de seleção do concurso de residência médica. Rev Bras Educ Med. 2006;30(3):115-124.

2. Grosseman S, Stoll C. O ensino-Aprendizagem da Relação Médico-Paciente:Estudo de Caso com estudantes do último semestre do Curso de Medicina. Rev Bras Educ Med.2008; 32(3):301-308.

3. Case SM, Swason DB. Constructing written test questions for the basic and clinical sciences. National Board of Medical Examiners. 2001; 9-180.

4. Case SM, Swason DB, Ripkey DR. Comparison of items in five-option and extended-matching formats for assessment of diagnostic skills. Acad. Med.1994;1-3.

5. Brasil. Ministério da Saúde. Guia de elaboração e revisão de itens: Instituto Nacional de Estudos e Pesquisas Educacionais Anísio Teixeira. Brasília; 2010.

6. Ferraz APCM, Belhot RV. Taxonomia de Bloom: revisão teórica e apresentação das adequações do instrumento para definição de objetivos instrucionais. 2010;17(2):421-431.
7. Pinto AC. Factores relevantes na avaliação escolar por perguntas de escolha múltipla. Psicologia, Educação e Cultura. 2001;5(1):23-44.

8. Almeida FN, Rouquayrol MZ. Introdução à Epidemiologia Moderna. Rio de Janeiro: APCE; 1990.

9. Padovani CR. Introdução à bioestatística. In: Campana AO. Introdução à investigação clínica. São Paulo: Trianon; 1995.

10. Instituto Brasileiro de Geografia e Estatística (IBGE). Normas de apresentação tabular. 3. ed. Rio de Janeiro: IBGE; 1993. $60 \mathrm{p}$.

11. Goodman LA. Simultaneous confidence intervals for constrasts among multinominal populations. Annals of Mathematical Statistics.1964;35(2):716-725.

12. Gonçalves A. Os testes de hipóteses como instrumental de validação da interpretação (estatística inferencial). In: Marcondes MA, Lakatos EM. Técnicas em Pesquisas. São Paulo: Atlas; 1982.

13. Moura ACS. FEF/UNICAMP, onde Saúde Coletiva e Performace Humana se encontram: uma pesquisa-ação. Campinas; 2006. Dissertação (Mestrado) — UNICAMP.

14. Costa NMSC. Docência no Ensino Médico: por que é tão difícil mudar? Rev Bras Educ Med.2007; 31(1):21-30.

15. Gomes R, Brino RF, Aquilante AG, Avó LRS. Aprendizagem baseada em problemas na formação médica e o currículo tradicional de Medicina: uma revisão bibliográfica. Rev Bras Educ Med.2009;33(3):444-451.

\section{CONTRIBUIÇÃO DOS AUTORES}

Aguinaldo Gonçalves concebeu, orientou e reviu o projeto em todas suas fases, além de formular e aplicar as questões. Eloá Rondi Bórnea procedeu à redação do texto e à fundamentação bibliográfica. Carlos Roberto Padovani executou o processamento e análises estatísticas.

\section{CONFLITO DE INTERESSES}

Declarou não haver.

\section{ENDEREÇO PARA CORRESPONDÊNCIA}

Aguinaldo Gonçalves

Av. John Boyd Dunlop, s/no

Jd. Ipaussurama - Campinas

CEP 13060-904 - SP

E-mail: aguinaldogon@uol.com.br 